\title{
Amniotic membrane-derived stem cells help repair osteochondral defect in a weight-bearing area in rabbits
}

\author{
ZHIJIN ZHANG ${ }^{1}$, LINRU ZENG ${ }^{2}$, JUN YANG ${ }^{1}$, LIN GUO $^{3}$, QIAO HOU ${ }^{1}$ and FANGBING ZHU ${ }^{4}$ \\ Departments of ${ }^{1}$ Hand Surgery and ${ }^{2}$ Foot and Ankle Surgery, Xiaoshan Hospital of Traditional Chinese Medicine, \\ Hangzhou, Zhejiang 311201; ${ }^{3}$ Department of Orthopaedics, Affiliated Zhongshan Hospital of \\ Dalian University, Dalian, Liaoning 116001; ${ }^{4}$ Department of Orthopaedics, Xiaoshan Hospital \\ of Traditional Chinese Medicine, Hangzhou, Zhejiang 311201, P.R. China
}

Received February 3, 2017; Accepted May 2, 2017

DOI: $10.3892 /$ etm.2017.4497

\begin{abstract}
Our study evaluated the use of amniotic membrane-derived stem cells for repairing osteochondral defects in a weight-bearing area in rabbits. Twenty-four 3-month-old male or female New Zealand white rabbits were selected. The rabbits were randomly divided into 3 groups of eight, according to the treatment received for an experimentally inflicted femoral medial malleolus lesion, group I received a human acellular amniotic membrane seeded with bone marrow-derived mesenchymal stem cells (HAAM-BMSCs) implant; group II received a simple HAAM implant and the control group received no experimental lesion or treatment. The rabbits were sacrificed at 12 and 24 weeks after the procedures (4 rabbits in each time-point) and the cartilage repair status in each animal was evaluated under the microscope. The tissue of the HAAM-BMSCs group grew well covering an area in the visual field that was significantly larger than that of the HAAM group $(\mathrm{p}<0.05)$. The percentage of collagen II-positive area in the HAAM-BMSC group was significantly higher than that in HAAM group $(\mathrm{p}<0.05)$. The number of chondrocytes determined by toluidine blue staining was higher in the HAAM-BMSC group than that in the HAAM group $(\mathrm{p}<0.05)$. The Wakitani scores of the HAAM and HAAM-BMSC groups were significantly higher (worse) than those of the normal control group ( $\mathrm{p}<0.05)$, but the score in the HAAMBMSC group was significantly lower than that in the HAAM group $(\mathrm{p}<0.05)$. The Wakitani scores in the HAAM-BMSC group were not different between the two time-points taken. Based on our findings, the amniotic membrane-derived stem cells had a good therapeutic effect in repairing the osteochondral defects in the weight-bearing area, and the number of chondrocytes in the injured area was increased significantly, which accelerated the repair of the damaged tissue in rabbits.
\end{abstract}

Correspondence to: Dr Zhijin Zhang, Department of Hand Surgery, Xiaoshan Hospital of Traditional Chinese Medicine, 152 Yucai Road, Xiaoshan, Hangzhou, Zhejiang 311201, P.R. China E-mail: zua6h4@163.com

Key words: amniotic membrane, stem cell, seeding cell resource

\section{Introduction}

Cartilage lesions are a common occurrence in Orthopedics due to trauma, osteochondritis, osteoarthritis and patellar softening. Studies of tissue-engineered cartilage have opened up new strategies for the restoration of articular cartilage. The lack of blood supply and innervation in the articular cartilage make injuries to these areas impossible to be spontaneously repaired (1). The joint trauma, infection and degenerative lesions caused by cartilage damage can lead to long-term joint pain and dysfunction, and even joint atrophy, leading to great pain and inconvenience. Traditional cartilage repair methods include microfracture surgery, subchondral drilling (2) and periosteal transplantation (3) with a purpose to induce the proliferation and differentiation of cells with chondrogenic potential for reconstruction. But those treatments usually only work well for fibrous cartilage (4) and the results in the repair of hyaline cartilage are poor and are often followed by further degradation and secondary ossification.

Autologous chondrocytes are currently the only tissueengineering cells used for clinical cartilage treatments. However, the proliferation of mature chondrocytes is difficult, the origin of autogenous cartilage is limited, and the extraction of autologous chondrocyte is inconvenient. In addition, in vitro differentiation can also easily occur, leading to the loss of cartilage formation ability. Furthermore, the activity of autologous chondrocytes decreases with age. Because of all these problems autologous chondrocytes usually fail to meet the requirements for tissue reconstruction $(1,2)$. Based on previous studies, we evaluated the application of amniotic membrane-derived stem cells as a seeding cell source to provide experimental evidence that could expand the repertoire of seeding cells available for cartilage tissue engineering.

\section{Materials and methods}

Reagents. The reagents used in the study included, fetal bovine serum (FBS; Institute of Biotechnology, Chinese Academy of Medical Sciences, Beijing, China); bovine serum albumin (BSA; Yuan Heng Sheng Ma Biotechnology Research Institute, Beijing, China); and high glucose DMEM (Gibco Life Technologies, Carlsbad, CA, USA) for cell culture. 
Type I collagenase (Gibco Life Technologies); polylysine (Sigma-Aldrich, St. Louis, MO, USA); immunohistochemical SP kit (Binyang, Tianjin, China); transferrin (Sigma-Aldrich); phenylmethylsulfonyl fluoride and Triton X-100 (both from Biodee Biotechnology, Beijing, China); trypsin (Huamei Biological Engineering Co., Ltd., Henan, China); and high glucose EDTA (Sigma-Aldrich) for sample processing. Insulin (Sigma-Aldrich); vitamin C (Invitrogen Life Technologies, Carlsbad, CA, USA); type II (Ab-1) mouse mAb and goat anti-mouse $\mathrm{IgG}(\mathrm{H}+\mathrm{L})$ (both from Merck Millipore, Billerica, MA, USA); and DAB coloring solution (Wuhan Boster Biological Engineering Co., Ltd., Wuhan, China) for other experiments.

Experimental equipment. An inverted phase contrast microscope (090-135.001; Leica, Wetzlar, Germany) was used for sample observations. The specialized equipment used to process and prepare the samples included a pressure steam sterilizer (Shanghai Boxun Industrial Co., Ltd., Shanghai, China), an automatic slicing machine (RM-2135; Leica), a constant temperature-type baking sheet machine (TK-218III; Hubei Taiwei Medical Technology Co., Ltd., Hubei, China), a biological tissue automatic dehydration machine (ZT-12H; Xiaogan Yaguang Medical-Electronic Technology, Hubei, China), and a biological tissue paraffin embedding machine (TB-718D; Hubei Taiwei Medical Technology, Co., Ltd.).

\section{Processing of experimental animals}

Grouping and handling. Twenty-four 3-month-old New Zealand white rabbits were selected without restriction on sex. Our study focused on the femoral medial malleolus from the 24 rabbits. The rabbits were divided into 3 groups of 8 rabbits each, according to the treatment provided to heal an experimental femoral medial malleolus lesion. Group I received an implanted human acellular amniotic membrane seeded with bone marrow-derived mesenchymal stem cells (HAAM-BMSCs) into the femoral condyle, group II a simple HAAM and the control group received no experimental lesion or treatment. The rabbits were sacrificed at 12 and 24 weeks after the procedure (4 rabbits in each time-point). Specimens from the knee joints were collected, processed, and stained with hematoxylin and eosin (H\&E) and toluidine blue and finally subjected to type II collagen immunohistochemical staining. Then the samples were subjected to Micro-CT and observation under inverted phase contrast microscope. This study was approved by the Animal Ethics Committee of Xiaoshan Hospital of Traditional Chinese Medicine.

Animal modeling. The rabbits were anesthetized with $3 \%$ pentobarbital sodium $(30 \mathrm{mg} / \mathrm{kg})$ injected through the ear vein. Under aseptic conditions, the skin of the bilateral knee joints was prepared and a medial incision was made on the medial region. Then, the joint capsule was cut along the quadriceps tendon medial edge. The patella was turned to reveal the femoral condyle and drill a hole with a 4-mm diameter drill to a depth of $3 \mathrm{~mm}$. According to the experimental design, a HAAM or HAAM-BMSCs complex was sutured to the medial femoral condyle. After the implant, the patella was reset and the tissue was suture-closed layer by layer. Finally, the animals were left to move freely and each of them was fed in a single cage.

\section{Handlings on BMSCs}

Isolation and culture. The rabbits were routinely disinfected in the operation area. A bone marrow puncture needle was used to penetrate into the proximal femur and tibia and $10 \mathrm{ml}$ syringe was connected to extract $4-5 \mathrm{ml}$ of bone marrow fluid. Next, the obtained bone marrow fluid was transferred into a centrifuge tube containing heparin and DMEM/F12. BMSCs were separated by whole bone marrow adherent separation and were then used for culture. Then bone marrow fluid was centrifuged at $1,050 \mathrm{xg}$ for $5 \mathrm{~min}$ at $4^{\circ} \mathrm{C}$, the supernatant was discarded and appropriate amount of DMEM/F12 was added to wash again for a second time. After centrifugation at $1,000 \mathrm{xg}$ for $5 \mathrm{~min}$ at $4^{\circ} \mathrm{C}$, the supernatant was discarded, and the cell number was counted and adjusted to a cell concentration of $5 \times 10^{5} / \mathrm{ml}$. FBS (15\%) and DMEM/F12 complete medium were added to make a cell suspension. The suspension was inoculated into a culture flask, and the flask was placed into an incubator for culture at $37^{\circ} \mathrm{C}$ with a $5 \% \mathrm{CO}_{2}$. The medium was completely replaced after $48 \mathrm{~h}$, and then every 48 or $72 \mathrm{~h}$. Seven or eight days later, when the cell fusion reached $85 \%$ confluence, the samples were digested with $0.25 \%$ trypsin-EDTA for no more than $30 \mathrm{sec}$ or $1 \mathrm{~min}$. A dilution ratio for subculturing of 1:2 or 1:3 was used. The FBS concentration was at $10 \%$ during successive culture transfers. The growth status of the cells was periodically observed and recorded under an inverted phase contrast microscope. BMSCs with uniform morphology, strong refraction and no granularity in their cytoplasm from the third generation were reserved for HAAM seeding.

Identification of BMSCs. Morphological observation. The morphology of adherent cells, their proliferation rate and population growth patterns were observed and photographed under an inverted phase contrast microscope.

\section{Specimen processing}

Evaluation of cartilage, tissue processing. The cartilage tissue from the rabbit models was taken for frozen sectioning. DAPI was used to stain nuclei, the survival of BMSCs after implantation was evaluated with fluorescence microscopy. In addition, all the samples were subjected to general observations, H\&E staining, toluidine blue staining and type II collagen immunohistochemical staining. The Wakitani scoring was used for histological scoring. The scoring criteria included the semi-quantitative analysis of cell type, medulla staining (metachromatic), surface integrity and thickness of cartilage. Scores ranged from 0 to 12 points. Lower scores indicate degrees of regenerative repair closer to the normal conditions (Table I).

Immunohistochemical staining of type II collagen. The specimens were fixed, decalcified and paraffin fixed. After sectioning, immunohistochemical staining of type II collagen was carried out as follows: i) tissue slides were placed in the oven at $50^{\circ} \mathrm{C}$ for $2 \mathrm{~h}$; ii) de-waxing was performed with 3 times washing using xylene, then hydration was performed by washing 4 times with 100, 90, 80 and $70 \%$ alcohol, respectively. Then the samples were washed 3 times with 
Table I. Modified Wakitani scores for cartilage defect evaluation.

\begin{tabular}{lll}
\hline Items & \multicolumn{1}{c}{ Criteria } & Value \\
\hline Cell type & Hyaline cartilage & 0 \\
& Most of them are hyaline cartilage & 1 \\
& Most of them are fibrocartilage & 2 \\
& Most of them are non-cartilage & 3 \\
& No cartilage & 4 \\
Medulla staining & Normal & 0 \\
(metachromatic) & Slight decrease & 1 \\
& Significantly reduced & 2 \\
& No change & 3 \\
Surface integrity & Smooth $(>3 / 4)$ & 0 \\
& Medium $(>1 / 2-3 / 4)$ & 1 \\
& Irregular $(1 / 4-1 / 2)$ & 2 \\
Thickness of & Severely irregular $(<1 / 4)$ & 3 \\
cartilage & $>2 / 3$ & 0 \\
Maximum & $1 / 3-2 / 3$ & 1 \\
total score & $<1 / 3$ & 2 \\
& & 3 \\
& &
\end{tabular}

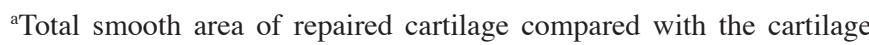
defect; ${ }^{b}$ the average thickness of the repaired cartilage compared with the thickness of the surrounding cartilage.

phosphate-buffered saline (PBS); iii) 1 volume $30 \% \mathrm{H}_{2} \mathrm{O}_{2}$ was mixed with 10 volumes of distilled water at room temperature for $10 \mathrm{~min}$ to inactivate endogenous enzymes and then the samples were washed 3 times with PBS; iv) a composite digestive solution was added and then left standing for $10 \mathrm{~min}$ at room temperature before washing 3 times with PBS; v) $5 \%$ BSA blocking solution was added and the samples slides were incubated at room temperature for $20 \mathrm{~min}$, to finally remove all excess liquid; vi) the primary antibody was diluted (mouse anti-rabbit type II collagen antibody) to $10 \mu \mathrm{g} / \mathrm{ml}$. The diluted antibody was added followed by incubation at $37^{\circ} \mathrm{C}$ for $2 \mathrm{~h}$, then slides were washed again with PBS 3 times for 3 min each; vii) a biotinylated goat anti-mouse IgG was added followed by incubation at $37^{\circ} \mathrm{C}$ for $20 \mathrm{~min}$, and then washing with PBS 3 times for $3 \mathrm{~min}$; viii) a streptavidin-biotin-peroxidase (SAB) complex was added followed by incubation at $37^{\circ} \mathrm{C}$ for $30 \mathrm{~min}$, and then washed with PBS 3 times for $5 \mathrm{~min}$; ix) for DAB color development, DAB was added and the samples were kept at room temperature for color development. Microscopic observation was used to control reaction time. Flushing with distilled water for $10 \mathrm{~min}$ terminated the reactions.

Hematoxylin staining was performed by covering slides with hematoxylin for $10 \mathrm{~min}$ and then flushing for $30 \mathrm{~min}$ with water. The samples were dehydrated by washing 3 times with 80,90 and $100 \%$ ethanol. Finally, the samples were made transparent by washing 3 times with xylene, 3 min for each time. Finally the samples were sealed with neutral gum for observation under the microscope.
Toluidine blue staining. Tissue sections were de-waxed and hydrated with conventional described methods above. The samples were kept in toluidine blue solution for $30 \mathrm{~min}$, and then gently washed with water. Next, samples were kept in glacial acetic acid solution until the nucleus and particles became clear, then gently washed with water and dried with cold air. Finally, the samples were treated with xylene to become transparent, and sealed with neutral gum.

Statistical analysis. The analysis software SPSS version 11 (IBM SPSS, Armonk, NY, USA) was used for statistical analyses. The gross and histological scores of the experimental and control groups at different time-points using the t-test were determined. A $\mathrm{P}<0.05$ was considered to be statistically significant.

\section{Results}

$H \& E$ staining of rabbit cartilage at 12 and 24 weeks. $\mathrm{H} \& \mathrm{E}$ staining of rabbit cartilage tissue in each group showed that tissue growth of HAAM-BMSCs group was better than that of HAAM group at both 12 and 24 weeks. The tissue coverage in the HAAM-BMSCs group was significantly higher than that of the HAAM group ( $p<0.05)$, and there was no significant difference between the HAAM-BMSCs and the control groups $(\mathrm{p}>0.05)$. H\&E staining images are shown in Fig. 1A.

Collagen II expression detected by immunohistochemical staining. Immunohistochemical staining was used to detect the expression levels of collagen II in each group. The percentage of collagen II-positive area in HAAM-BMSCs group was significantly higher than that in HAAM group $(\mathrm{p}<0.05)$, and there was no significant difference between the HAAM-BMSCs and the control groups ( $\mathrm{p}>0.05)$ (Fig. 2).

Toluidine blue staining at 12 and 24 weeks. In order to detect the expression of DNA in rabbit cartilage tissue, we used toluidine blue staining to detect the number of stained chondrocytes in the HAAM-BMSC group. The number of condrocytes in HAAM-BMSC group was significantly higher than that in HAAM group $(\mathrm{p}<0.05)$, and there was no statistically significant difference between the number in the HAAM-BMSCs and that in the control group ( $\mathrm{p}>0.05)$ (Fig. 3).

Wakitani scoring of histological cartilage defect. The Wakitani scoring for histological cartilage defects was used to further evaluate tissue repair in each group. The Wakitani scores of the HAAM and HAAM-BMSC groups were both significantly higher than those of the control group $(\mathrm{p}<0.05)$. However, compared with the HAAM group, the Wakitani score of the HAAM-BMSCs was significantly lower $(\mathrm{p}<0.05)$ but still higher than that of the normal control group $(\mathrm{p}<0.05)$. At 24 weeks, the Wakitani score in group I was significantly lower compared with the score at 12 weeks $(\mathrm{p}<0.05)$. There was no significant difference in Wakitani score in the HAAM-BMSCs group between the 2 time-points (Table II).

\section{Discussion}

Articular cartilage injuries do not get well repaired spontaneously, and are often the harbingers of degenerative arthritis, 
A

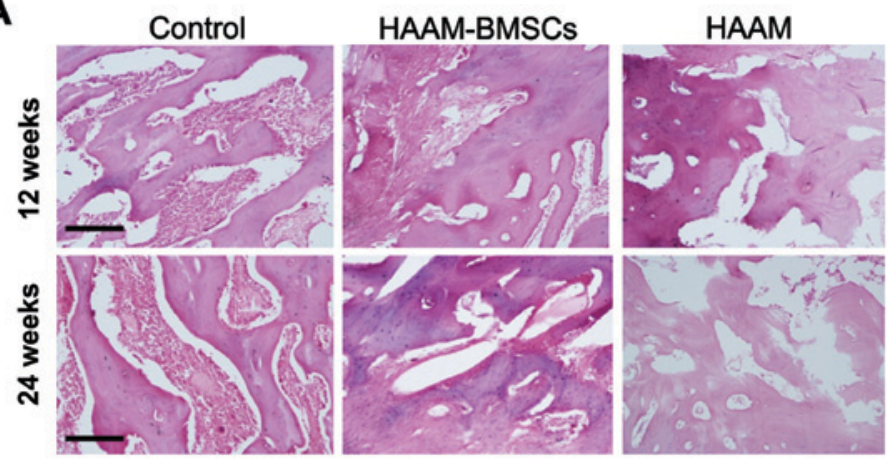

B

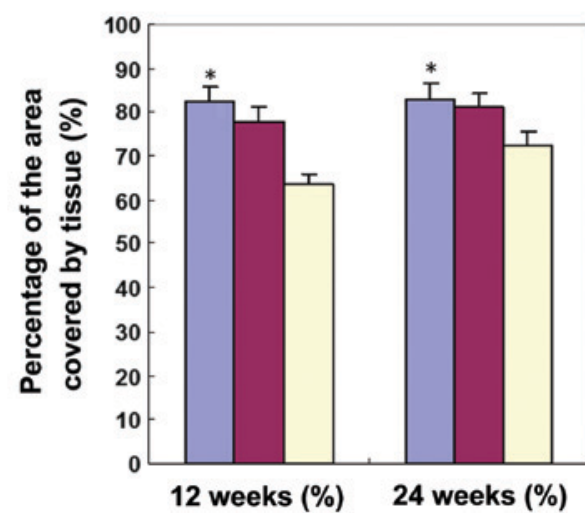

Fe the cartilage between the three groups. (A) H\&E staining, x100, inverted phase contrast microscopy (B) Compared with HAAM group, HAAM-BMSC group had better tissue growth. The area of tissue coverage was significantly higher in the HAAM-BMSC group than that in HAAM group at both time-points, and the difference was statistically significant $\left({ }^{*} \mathrm{p}<0.05\right)$. There was no significant difference between HAAM-BMSCs and the control group ( $>0.05$ ). HAAM, human acellular amniotic membrane; BMSCs, bone marrow-derived mesenchymal stem cells.

A
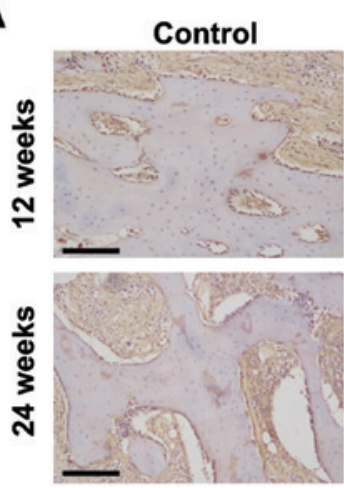

B

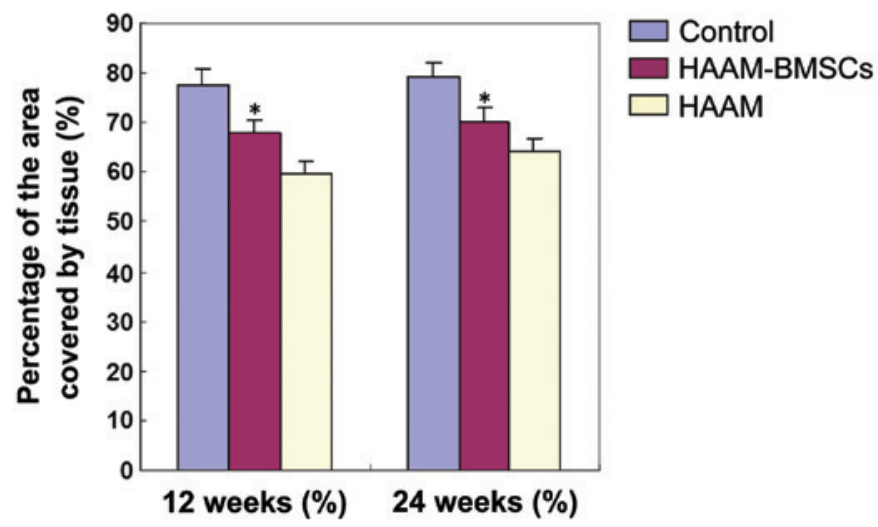

HAAM-BMSCs
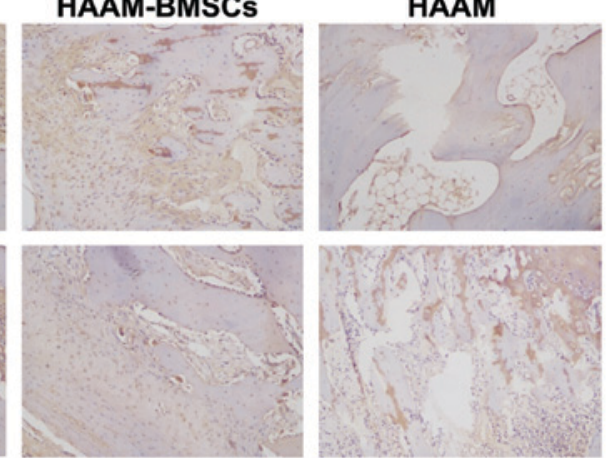

$\square$ Control

HAAM-BMSCs

$\square$ HAAM

Figure 2. Immunohistochemical staining of cartilage tissue. (A) Immunohistochemical staining of cartilage tissue slides at 12 and 24 weeks (x100, inverted phase contrast microscopes). (B) The presence of collagen II in the group II (HAAM-BMSCs) was more anbundant than that of the HAAM group at both 12 and 24 weeks, and the difference was statistically significant $\left({ }^{*} \mathrm{p}<0.05\right)$. There was no significant difference between the HAAM-BMSCs and the control group ( $>0.05$ ). HAAM, human acellular amniotic membrane; BMSCs, bone marrow-derived mesenchymal stem cells. 
A
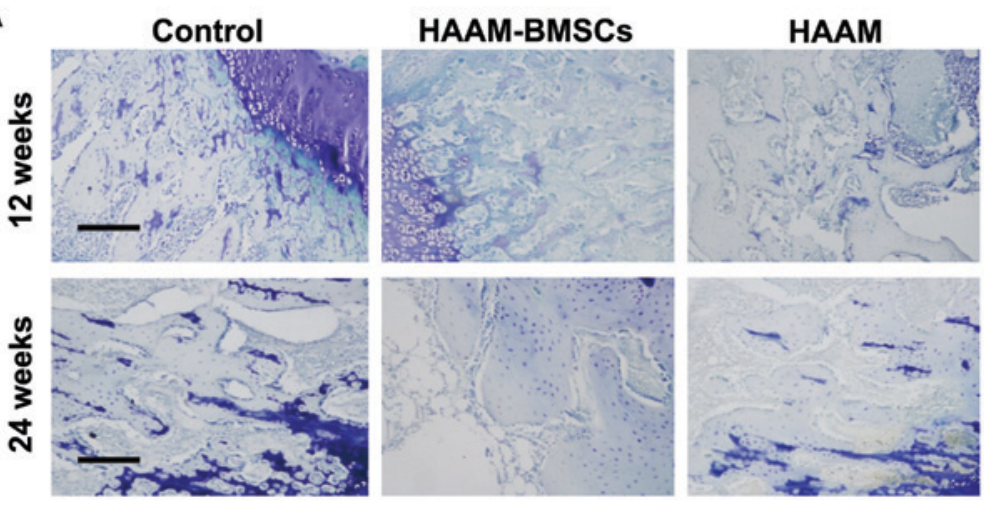

B
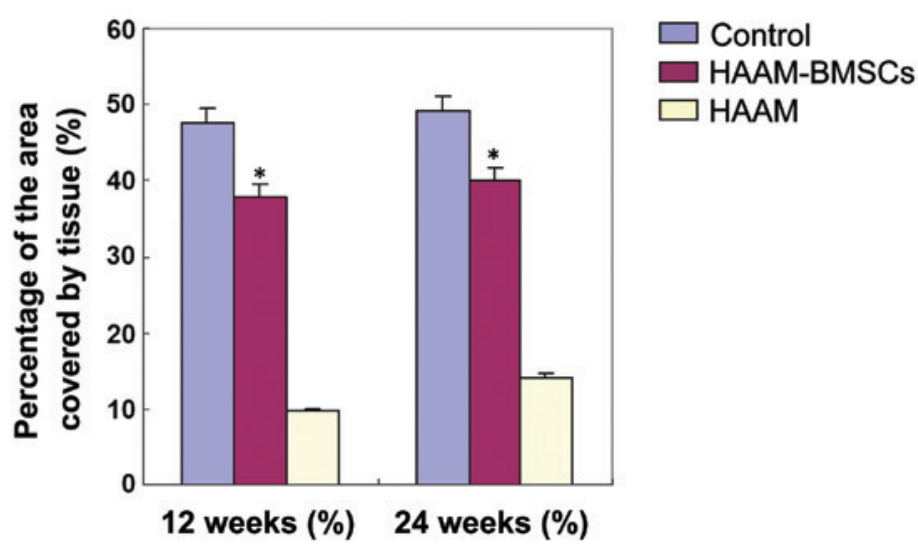

mine staining of cartilage lesion. (A) Toluidine blue staining of cartilage lesion slides at 12 and 24 weeks (x100, inverted phase contrast higher than that in HAAM group ( ${ }^{*} \mathrm{p}<0.05$ ), but there was no statistically significant difference between HAAM-BMSCs and the control group (p $>0.05$ ). HAAM, human acellular amniotic membrane; BMSCs, bone marrow-derived mesenchymal stem cells.

Table II. Wakitani scoring of histological cartilage defects.

\begin{tabular}{lcccc}
\hline Groups & 12 weeks (\%) & 24 weeks (\%) & T-value & P-value \\
\hline Control group & $0.00 \pm 0.01$ & $0.00 \pm 0.01$ & 0.43 & 0.52 \\
HAAM (group I) & $10.38 \pm 0.21$ & $9.47 \pm 1.11$ & 0.44 & 0.57 \\
$\begin{array}{l}\text { HAAM-BMSCs } \\
\text { group II) }\end{array}$ & $6.33 \pm 0.38^{\mathrm{a}}$ & $3.05 \pm 1.28^{\mathrm{a}}$ & 1.28 & 0.03 \\
& & & &
\end{tabular}

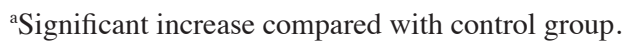

eventually leading to joint dysfunction. Therefore, interventions to repair damaged cartilage in order to avoid or delay the occurrence of secondary osteoarthritis are actively sought by researchers. In recent years, developments in tissue engineering for cartilage repair have widened the prospects. The selection of seeding cells is a prerequisite and key to the success of tissue engineering. In most cases, BMSCs were used as seeding cells (2). However, due to the limited amounts of bone marrow for cell extraction, and the fact that the number of stem cells decreases gradually with age, the use of BMSCs is not without problems (3).

Amniotic MSCs, with their strong in vitro proliferation and differentiation capabilities are easy to obtain in large quantities with only slight damage to the donor site. Therefore, adipose-derived MSCs have become a new source of tissue engineering seeding cells and gene therapy target cells. Besides the nature of seeding cells, the local presence of cytokines is another important factor that can promote the formation of cartilage locally (4). In our study, bone marrow-derived stem cells were harvested by mechanical isolation, enzymatic digestion and centrifugation. We confirmed the identity of the stem cells, and proved they could differentiate into adipocytes and chondrocytes.

The space between cartilage cells is filled with cartilage matrix, which is the scaffold of cartilage tissue. The cartilage matrix is indispensable to maintain the biomechanical characteristics of articular cartilage. It reduces the friction coefficient, buffers mechanical shock and mediates the signal transmission between cells $(5,6)$. For tissue engineering of cartilage, a cartilage matrix has the ideal three-dimensional structure, physical and chemical properties, and good biocompatibility with bone marrow-derived stem cells (7), making it a reliable stem cell carrier. In the process of inducing stem cell differentiation into chondrocytes, the cartilage matrix plays 3 different roles, it is a single-cell factor (8); a combined cytokine (9); and a continuous cytokine (10).

Amniotic membranes contain immature undifferentiated cells because they form in the early stages of embryogenesis. Amniotic membrane cells are amniotic epithelial cells, fibroblasts and undifferentiated mesenchymal cells. Fibroblasts can produce collagen fibers, elastic fibers, reticular fibers, a variety of glycoproteins, proteoglycans, collagen, 
hyaluronic acid and fibronectin $(11,12)$. Mesenchymal cells have the potential to differentiate during inflammation and can do so into dendritic cells and fibroblasts. Amniotic epithelial cells in vitro can express glial fibrillary acidic protein and liver parenchymal cell differentiation markers, albumin and $\alpha$-fetoprotein, which indicates that they can be seeding cells for nerve or liver tissue engineering (13). Amniotic epithelial cells rarely express human leukocyte antigens $\mathrm{A}, \mathrm{B}$ and $\mathrm{C}$ or DR or other antigens and therefore their transplantation does not usually lead to immune rejection. Studies have been carried out to explore the use of amniotic epithelial cells to treat nerve damage, amniotic membrane premature rupture, and corneal epithelial defects (14-16). Thus, the use of amniotic epithelial cells for tissue engineering may lead to successful outcomes.

In a study, human embryonic periosteum-derived osteoblasts and human amniotic cells were combined in vitro to construct a tissue engineered periosteum with the ability to promote differentiation of new osteoblasts, and increase expression levels of Cbfa1 (17). As a scaffold material, human amniotic membrane can induce and promote osteoblast differentiation. Tissue engineered periosteum constructed by the combination of osteoblasts and human amniotic membrane has been used for production of bone tissue at a molecular level, where the proliferation of condrocytes was evidenced (18). The amniotic membrane has showed good ability for water absorption, cell adsorption and biocompatibility in vitro. Condylar cells can adhere and proliferate on the amniotic membrane. The amniotic membrane has been proposed as a scaffold for condylar tissue engineering (19-22).

In our study, we evaluated the use of amniotic-loaded stem cells to repair a skeletal cartilage lesion, by implanting a BMSC-HAAM complex into an injured medial condyle of rabbits. At 12 and 24 weeks after implantation, the number of chondrocytes, the collagen II expression level and the Wakitani scores in the medial condylar cartilage of the BMSC-HAAM group were all significantly better than those in the HAAM group. The results suggest that amniotic membrane-loaded stem cells have a good reparation effect on the damaged medial condyle of rabbits, which is consistent with the results of previous studies. We believe that the application of amniotic membrane-loaded stem cells, as a tissue engineering repair material, has good clinical prospects.

Based on our findings, the amniotic membrane-derived loaded stem cell repair strategy used had a good therapeutic effect on the osteochondral defect, in the weight-bearing area of the femoral medial malleolus. The number of chondrocytes in the injured area increased significantly with the HAAM-BMSC treatment, which accelerated the repair of damaged tissue in rabbits. For clinical experiments in humans further experiments and ethical aspects still need to be considered.

\section{References}

1. Takao M, Uchio Y, Kakimaru H, Kumahashi N and Ochi M Arthroscopic drilling with debridement of remaining cartilage for osteochondral lesions of the talar dome in unstable ankles. Am J Sports Med 32: 332-336, 2004.

2. Mithoefer K, Williams RJ 3rd, Warren RF, Potter HG, Spock CR, Jones EC, Wickiewicz TL and Marx RG: The microfracture technique for the treatment of articular cartilage lesions in the knee. A prospective cohort study. J Bone Joint Surg Am 87: 1911-1920, 2005
3. Grande DA, Breitbart AS, Mason J, Paulino C, Laser J and Schwartz RE: Cartilage tissue engineering: current limitations and solutions. Clin Orthop Relat Res 367 (Suppl): S176-S185, 1999.

4. Enosawa S, Sakuragawa N and Suzuki S: Possible use of amniotic cells for regenerative medicine. Nihon Rinsho 61: 396-400, 2003 (In Japanese).

5. Okawa H, Okuda O, Arai H, Sakuragawa N and Sato K: Amniotic epithelial cells transform into neuron-like cells in the ischemic brain. Neuroreport 12: 4003-4007, 2001.

6. Bilic G, Ochsenbein-Kölble N, Hall H, Huch R and Zimmermann R: In vitro lesion repair by human amnion epithelial and mesenchymal cells. Am J Obstet Gynecol 190: 87-92, 2004.

7. Parmar DN, Alizadeh H, Awwad ST, Li H, Neelam S, Bowman RW, Cavanagh HD and McCulley JP: Ocular surface restoration using non-surgical transplantation of tissue-cultured human amniotic epithelial cells. Am J Ophthalmol 141: 299-307, 2006

8. Pollok JM and Vacanti JP: Tissue engineering. Semin Pediatr Surg 5: 191-196, 1996.

9. Eyrich D, Wiese H, Maier G, Skodacek D, Appel B, Sarhan H, Tessmar J, Staudenmaier R, Wenzel MM, Goepferich A, et al: In vitro and in vivo cartilage engineering using a combination of chondrocyte-seeded long-term stable fibrin gels and polycaprolactone-based polyurethane scaffolds. Tissue Eng 13: 2207-2218, 2007.

10. Liu Y, Shu XZ and Prestwich GD: Osteochondral defect repair with autologous bone marrow-derived mesenchymal stem cells in an injectable, in situ, cross-linked synthetic extracellular matrix. Tissue Eng 12: 3405-3416, 2006.

11. Freed LE, Vunjak-Novakovic G, Biron RJ, Eagles DB, Lesnoy DC, Barlow SK and Langer R: Biodegradable polymer scaffolds for tissue engineering. Biotechnology 12: 689-693, 1994.

12. Mahgoub MA, Ammar A, Fayez M, Edris A, Hazem A, Akl M and Hammam O: Neovascularization of the amniotic membrane as a biological immune barrier. Transplant Proc 36: 1194-1198, 2004

13. Kim BS and Mooney DJ: Development of biocompatible synthetic extracellular matrices for tissue engineering. Trends Biotechnol 16: 224-230, 1998.

14. Burg KJ, Porter S and Kellam JF: Biomaterial developments for bone tissue engineering. Biomaterials 21: 2347-2359, 2000.

15. Nakamura T and Kinoshita S: Ocular surface reconstruction using cultivated mucosal epithelial stem cells. Cornea 22 (Suppl 7): S75-S80, 2003.

16. Nakamura T, Endo K, Cooper LJ, Fullwood NJ, Tanifuji N, Tsuzuki M, Koizumi N, Inatomi T, Sano Y and Kinoshita S: The successful culture and autologous transplantation of rabbit oral mucosal epithelial cells on amniotic membrane. Invest Ophthalmol Vis Sci 44: 106-116, 2003.

17. Ducy P, Starbuck M, Priemel M, Shen J, Pinero G, Geoffroy V, Amling M and Karsenty G: A Cbfa1-dependent genetic pathway controls bone formation beyond embryonic development. Genes Dev 13: 1025-1036, 1999.

18. Leyva-LeyvaM,Barrera L,López-CamarilloC,Arriaga-Pizano L, Orozco-Hoyuela G, Carrillo-Casas EM, Calderón-Pérez J, López-Díaz A, Hernandez-Aguilar F, González-Ramírez R, et al: Characterization of mesenchymal stem cell subpopulations from human amniotic membrane with dissimilar osteoblastic potential. Stem Cells Dev 22: 1275-1287, 2013.

19. Inatomi T, Nakamura T, Koizumi N, Sotozono C and Kinoshita S: Current concepts and challenges in ocular surface reconstruction using cultivated mucosal epithelial transplantation. Cornea 24 (Suppl 8): S32-S38, 2005.

20. Nakamura T, Inatomi T, Sotozono C, Amemiya T, Kanamura N and Kinoshita S: Transplantation of cultivated autologous oral mucosal epithelial cells in patients with severe ocular surface disorders. Br J Ophthalmol 88: 1280-1284, 2004.

21. Inatomi T, Nakamura T, Koizumi N, Sotozono C, Yokoi N and Kinoshita S: Midterm results on ocular surface reconstruction using cultivated autologous oral mucosal epithelial transplantation. Am J Ophthalmol 141: 267-275, 2006.

22. Ti SE, Grueterich M, Espana EM, Touhami A, Anderson DF and Tseng SC: Correlation of long term phenotypic and clinical outcomes following limbal epithelial transplantation cultivated on amniotic membrane in rabbits. Br J Ophthalmol 88: 422-427, 2004. 